\title{
Влияние стратегических рисков на финансовые результаты компании
}

\author{
Черкасова В.А. ${ }^{27}$, Батенкова А.А. ${ }^{28}$
}

В статье раскрывается концептуальное представление о риске со стратегической точки зрения. Выявляются основные стратегические риски компании и подходы, связанные с их оченкой. Предлагается модель, измеряющая стратегический риск через вероятность потери положения компании в рейтинге среди других фирм.

Почему экономические агенты, которые в большинстве своем не склонны к риску, вообще подвергают себя ему? Одной из причин, вероятно, выступает то, что они верят в возможность использования рисков в качестве ресурса для создания и преувеличения прибыли и рассчитывают на рост стоимости компании. Иначе как можно было бы, например, объяснить стремление крупных международных компаний вторгаться на развивающиеся рынки в страны, рейтинг политической стабильности которых низок и не собирается улучшаться. Например, индекс политической стабильности России (DESIX), рассчитываемый Eurasia Group, составил в 2005 году 58 пунктов из 100, это примерно уровень Филиппин, Аргентины, Турции. И, несмотря на это, в нашу страну продолжают вливаться инвестиции из-за границы, а международные компании стремительно осваивают наш рынок. Крупнейшие автомобильные концерны, такие как Форд, Нисан, Крайслер, строят заводы в городах России. Авиастроительные компании мирового уровня заключают договоры о производстве деталей для своих самолетов в России... Список рискованных шагов, предпринимаемых предприятиями ежедневно, можно продолжить до бесконечности. General Motors в 20-e, IBM в 50e, Microsoft и Intel в 80-е и Google сегодня, достигли своего успеха не избегая риска, а, наоборот, выискивая его. Суть их успеха в способности управлять риском. Сегодня ни одна компания, нацеленная на процветание, не обходится без услуг риск-менеджера, что оправдывает современные тенденции, заключающиеся в отношении к риску как к неотъемлемой компоненте любого бизнеса. Несмотря на то, что тема риска не является новой для экономистов и начала активно освещаться в литературе ещё около 50 лет назад, ученые до сих пор расходятся во мнениях относительно определения рисков, их классификации, измерения и оптимизации. Более того, сомнительно, что экономическая наука вообще когда-нибудь придет к четкому разделению рисков: очевидно, что множество предложенных классификаций и моделей имеют право на существование. Риски многомерны и их система сложна и разветвлена, но, несмотря на это, всё новые и новые типы рисков выделяются и становятся предметом активных исследований и дискуссий экономической науки. Одним из таких ещё пока малоизученных рисков выступает стратегический риск.

\footnotetext{
${ }^{27}$ К.э.н, доцент кафедры экономики и финансы фирмы ГУ-ВШЭ

${ }^{28}$ Студентка факультета экономики ГУ-ВШЭ
}

Выпуск \#3, 2007

(C) Электронный журнал Корпоративные Финансы, 2007 
Понятие стратегического риска неотъемлемо связано с понятием стратегии. Иначе, стратегический риск можно определить, как риск, связанный со стратегией. Такой риск угрожает способности управляющих своевременно и качественно разрабатывать стратегию управления фирмой и внедрять её [R. Simons, 1999]. Чтобы лучше разобраться в понятии этого риска, необходимо четко осознавать, что собой представляет понятие самой стратегии. Это поможет определить сферы влияния изучаемого нами риска, его типы и особенности.

Несмотря на, то, что понятие стратегии на несколько десятков лет старше понятия стратегического риска, единства теорий нет и здесь. Ученые и практики используют это понятие свободно, и никто не спорит о существовании стратегии и её огромной значимости в сфере бизнеса. Поэтому в данной статье сосредоточимся на теоретических подходах к определению стратегии Chaffee и выделяющего три типа стратегий. Прежде чем приступить к описанию этого подхода, интересно заметить, что в реальной бизнес среде менеджеры, вообще говоря, весьма смутно представляют себе, что такое стратегия. В исследовании, проведенном Неini Inkavalko и Petri Aaltonen, учеными из Хельсинского Университета Технологий, было выявлено, что около 50\% менеджеров среднего звена определяют стратегию как последовательность действий, ассоциируя со стратегией понятия политики, управленческого принципа и схемы действий [Heini Inkavalko, Petri Aaltonen, 2001]. При этом наиболее популярным ответом среди менеджеров высшего звена было определение стратегии как цели и направления действий.

В теории экономисты исходно придерживаются базовой предпосылки, что стратегия связана с нераздельностью фирмы и окружающей её среды [Lenz, 1980]. Фирма прибегает к стратегии как к средству, помогающему выживать в изменяющейся среде. Именно из-за того, что эти изменения постоянно проявляются в виде различных комбинаций обстоятельств, сама стратегия остается незапрограммированной, нерутинной и неповторяющейся. Соответственно, стратегические решения также в целом не являются рутинами, но при этом крайне важны, так как влияют на общее благосостояние предприятия. Неявно все экономисты соглашаются, что стратегия включает в себя, как и сам смысл действий, постепенно реализующихся фирмой, то есть «содержание стратегии», так и процесс реализации этих действий. Более того, планируемые, находящиеся в процессе реализации и реализованные в итоге стратегии могут не иметь ничего общего между собой. Chaffee выделяет существование трех групп определений стратегии в экономической литературе [Chaffee, 1985] .

Первая модель - линейная- концентрируется на планировании. Она опирается на классическое определение стратегии, сформулированное Chandler в 1962 году: стратегия - определение базовых долгосрочных иелей предприятия, и выбор курса действий и распределение ресурсов, необходимые для достижения этих иелей [Chandler, 1962]. В соответствии с линейной моделью, стратегия состоит из интегрированных решений, действий, планов, которые определят и помогут достичь поставленных целей организации. И цели, и способы их достижения являются результатами соответствующих стратегических решений. Для достижения этих целей фирма устанавливает и изменяет свои связи с окружающей её средой, варьируя предоставляемые потребителю продукты и выходя на различные рынки, а также реализуя другие предпринимательские решения. Линейная модель подразумевает, что топ менеджеры могут влиять на деятельность организации. Окружающая фирму среда неявным образом выступает для них некой «неприятностью», которая главным образом состоит из конкурентов. Топ менеджеры определяют свои цели, конструируют альтернативные методы их достижения, взвешивают вероятность их успешной реализации и потом решают, какой из этих методов воплотить в реальность. В процессе выполнения выбранного 
метода, менеджеры получают дополнительную прибыль от наступления в будущем благоприятных событий и тенденций и пытаются избежать или противостоять нежелательным. Модель изначально была разработана для бизнеса нацеленного на прибыль, а, следовательно, измерителями результативности стратегии выступают прибыль и продуктивность.

Процесс стратегического планирования, по мнению Lorange and Vancil, концептуально прост: менеджеры на каждом уровне иерархии, в конце концов, приходят к единому мнению относительно детального интегрированного плана действий на будущий год; они начинают с выработки корпоративных целей и заканчивают одно или двух годичным планом прибылей [Lorange P., Vancil R.F., 1978]. Если предприятие хочет, чтобы план последовательных действий был успешно реализован на практике, организация должна быть едина, то есть, способна к тому, чтобы решения принятые сверху могли быть реализованы на нижних уровнях. Только в этом случае намерения станут действиями.

Второе предположение линейной модели исходит из предсказательной сути планирования: решения, предпринятые сегодня, основаны на вере в определенные суждения о будущих состояниях природы, которые могут не наступить в течение месяцев, годов и даже десятков лет. И для того чтобы планирование не было бессмысленным мы должны предполагать, что, либо будущее все-таки в какой-то мере предсказуемо, или то, что фирма изолирована или защищена от окружающей среды настолько, что будущие состояния природы не важны для нас. Авторы, придерживающиеся стратегии в рамках линейной модели, сходятся на том, что фирмы имеют цели, а достижение этих целей - самый главный результат стратегии.

Следующая модель, которую выделял Chaffee, адаптивная модель. Модель исходит от определения стратегии, данного Hofer: «стратегия связана $c$ разработкой жизнеспособных соответствий между возможностями и рисками, предоставляемыми внешней средой, и наличием у фирмы способностей и ресурсов для использования этих возможностей» [Hofer, 1973]. Предприятие должно постоянно оценивать внешние и внутренние условия. За оценкой следует приспособление фирмы к этим выявленным изменениям, что в конечном итоге обеспечит удовлетворительную расстановку сил между возможностями и рисками среды, с одной стороны, и возможностями и ресурсами фирмы, с другой. Линейная модель концептуально отличается от адаптивной следующими параметрами:

1.Мониторинг окружающей организацию среды и внесение последующих изменений в действия организации выполняются постоянно и одновременно в соответствии с адаптивным подходом к стратегии.

2.Адаптивная концепция не имеет такого тесного взаимодействия с решениями относительно будущих целей. Своеобразной целью здесь выступает поддержание здоровой ежемоментной связи между организацией и средой, которая бы приносила удовлетворительные результаты.

3.Адаптивная модель идет дальше в своем определении, чем линейная, так как концентрируется не только на изменении решений относительно продуктов и рынков, а также затрагивает некие изменения в стиле, маркетинге, качестве и других нюансах.

4.Для адаптивной стратегии относительно неважно долгосрочное планирование, а как следствие разработка решений о стратегии менее централизована и более многогранна.

5.В соответствии с адаптивной концепцией окружающая фирму среда выступает более сложным организмом, состоящим из тенденций, событий, конкурентов, акционеров. Как следствия фирма и окружающая среда более 
открыты друг другу. Окружающая среда едва поддаётся долгосрочному планированию. Соответственно фирма должна не столько «иметь дело со средой», сколько «изменяться со средой».

Третья модель - интерпретащионная, ассоциируется с социальными и культурными аспектами предприятия. Реальность в рамках такого подхода к стратегии социально окрашена, и отнюдь не является чем-либо объективным и экзогенным, которое может быть правильно или неправильно истолковано. Напротив, реальность определяется через процесс обмена восприятиями между взаимодействующими объектами, в котором эти восприятия укрепляются, изменяются или исчезают в соответствии с их согласованностью с восприятиями других агентов. Стратегия в рамках интерпретационной модели может быть определена как метафоры-ориентиры и рамки, которые позволяют фирме и среде быть понятыми друг другом и акционерами. Акционеры должны быть мотивированы поступать таким образом, чтобы это было благоприятно для фирмы. Одно из главных отличий интерпретационного подхода от адаптивного в том, что стратегия фирмы заключается не в изменении со средой, а во взаимодействии со средой, что как раз характерно для линейной модели. Линейная стратегия призвана иметь дело со средой посредством организационных решений, интерпретационная же воздействует на среду символами и коммуникацией. Интерпретационная стратегия также как и адаптивная воспринимает фирму и среду как открытые системы. Но в соответствии с интерпретационной моделью руководство предприятие стремится сформировать потенциальное отношение участников взаимодействия к своему бизнесу и продукции; они не производят постоянных физических изменений в этой продукции. Целью выступает повышение доверия к себе. Такая стратегия более активна, чем адаптационная, которая направлена на постоянную подстройку к изменяющейся среде.

Можно подвести итог, определив стратегии следующим образом. В соответствии с линейным подходом, руководство предприятия планируют, как они будут взаимодействовать с конкурентами с целью достижения долгосрочных целей, выраженным в форме прибыли и стоимости компании. По адаптивной концепции, фирма и её составляющие постоянно меняются, чтобы подстроиться под требования окружающей среды и под предпочтения потребителей. Интерпретационная стратегия нацелена на формирование отношения к фирме участников и потенциальных участников взаимодействия. Очевидно, что невозможно относиться к представленным подходам как к абсолютно различным, непересекающимся между собой и взаимно исключаемым. К тому же они хорошо отражают постепенную эволюцию понятия стратегия от простого линейного подхода к более замысловатому и многогранному интерпретационному, и дают первоначальное представление о возможных источниках стратегических рисков.

Определив смысл понятия «стратегия» становится проще определить понятие «стратегического риска». Итак, под стратегическим риском можно подразумевать риски, связанные, во-первых, с выработкой неправильных, неадекватных, неподходящих к данной окружающей среде планов и моделей поведения фирмы; во-вторых, к стратегическим же рискам будут относиться риски, связанные с наступлением каких либо внешних событий, которые сделают невозможным реализацию данной стратегии, сделают её неадекватной и потребуют либо её модификации, либо её замены на новую стратегию; третий аспект стратегического риска состоит в неправильной реализации стратегии, даже при условии, что сама стратегия выработана адекватно, а внешние условия стабильны. Говоря о стратегических рисках, безусловно, больший упор делается на долгосрочный период и на долгосрочные цели компании, однако в том смысле, в котором текущие действия и решения влияют на долгосрочную перспективу, они также 
считаются стратегическими, а, следовательно, сопряжены со стратегическими рисками.

Рассмотрим положение стратегических рисков относительно других ключевых рисков, угрожающих любому бизнесу. Среди наиболее важных рисков выделяют финансовые риски, операционные риски, моральные риски и изучаемые нами стратегические риски. Важно, что составляющие этих рисков могут проявляться как извне, так и изнутри. Пример разделения факторов несущих риск извне и изнутри приведен в таблице 1.

Таблица 1 - Области проявления рисков

\begin{tabular}{|l|l|l|}
\hline \multicolumn{1}{|c|}{ Виды рисков } & Проявляющиеся извне & \multicolumn{1}{|c|}{ Проявляюшиеся изнутри } \\
\hline Финансовые риски & $\begin{array}{l}\text { Риски процентной ставки } \\
\text { Риски обменного курса } \\
\text { Кредитные риски }\end{array}$ & $\begin{array}{l}\text { Риски ликвидности и } \\
\text { денежных потоков }\end{array}$ \\
\hline $\begin{array}{l}\text { Стратегические } \\
\text { риски }\end{array}$ & $\begin{array}{l}\text { Конкуренция } \\
\text { Изменение предпочтений } \\
\text { потребителя } \\
\text { Отраслевые сдвиги } \\
\text { Изменение спроса }\end{array}$ & $\begin{array}{l}\text { Исследования и разработки } \\
\text { Интеллектуальная } \\
\text { собственность }\end{array}$ \\
\hline Операционные риски & $\begin{array}{l}\text { Управление } \\
\text { Культура } \\
\text { Директора }\end{array}$ & $\begin{array}{l}\text { Системы бухгалтерского учета } \\
\text { Информационные системы } \\
\text { Рекрутмент } \\
\text { Поставки } \\
\text { Производственный процесс }\end{array}$ \\
\hline Моральные риски & Поставщики & $\begin{array}{l}\text { Работники } \\
\text { Собственность } \\
\text { Продукты и услуги }\end{array}$ \\
\hline
\end{tabular}

Наиболее современная классификация стратегических рисков принадлежит J.Slywotzky и John Drzik [J.Slywotzky и John Drzik, 2005] . Они выделяют 7 основных классов стратегических рисков:

1.Риски, связанные с отраслью (снижение прибыльности, повышение требуемых затрат на R\&D, избыток производственных мощностей, дерегуляция, повышение власти поставщиков, чрезмерная волатильность бизнес циклов)

2.Риски, связанные с технологией (сдвиг в технологиях, истечение срока патента, устаревание производственного процесса)

3.Риски, связанные с брендом (эрозия, коллапс)

4.Риски, связанные с конкурентами (вхождение новых крупных игроков, захват конкурентом крупной рыночной доли, идентичный вашей фирме конкурент)

5.Риски, связанные с потребителями (сдвиг в предпочтениях потребителей, увеличение власти потребителей, чрезмерная зависимость от узкого круга потребителей)

6.Риски, связанные с проектами (провал $\mathrm{R} \& \mathrm{D}$, провал IT, провал развития нового бизнеса, провал слияния или поглощения)

7. Риск стагнации бизнеса (отсутствие роста выпуска или спад, рост выпуска, сопутствующий падением цен, отсутствие новой продукции)

Из классификации видно, что сюда относятся, как и внешние риски, так и внутренние. Реализация любого из перечисленных рисков может привести к серьезным последствиям для предприятия, и потребует разработки новых стратегических решений, что очень сложно и дорого. При этом если рассуждать в терминах определения стратегического риска, сформулированного в работе ранее, 
получается, что эти риски, с одной стороны, могут быть сгенерированы непосредственно неправильно выработанной стратегией, с другой стороны, неаккуратным её воплощением в жизнь. Также осуществление стратегических рисков может привести к тому, что стратегию придется модифицировать или же разрабатывать новую, что в нашем определении отражало третий аспект стратегического риска. Однако, стоит заметить, что эти аспекты также пересекаются между собой: очевидно что фирмы должны стремиться вырабатывать такие стратегии, которые бы были устойчивы к стратегическим рискам и даже более того, не провоцировали бы их. Из этого следует, что, вообще говоря, сложно провести грань между тем, когда стратегический риск действительно возникает экзогенно, и в этом нет вины предприятия, или же, когда он возникает эндогенно, вследствие неадекватной стратегии. В рамках такого подхода к рискам, очевидно, что финансовые, операционные и моральные риски также могут иметь стратегический характер, если масштаб их влияния настолько серьезен, что приведет к провалу стратегии. Например, если на фабрике по производству автомобилей будет произведена партия бракованных машин новой модели, и дело в будущем получит широкое освещение в прессе, что приведет к оттоку клиентов и коллапсу бренда, то реализованный риск, безусловно, относится к ряду стратегических. Хотя классически брак относят к операционным рискам. Таким образом, стратегические риски не столько образуют отдельный класс рисков, сколько формируют концептуально иной подход к анализу рисков бизнеса.

Оценка рисковой позиции крайне важна для экономики, она влияет на цели и положение экономических агентов в системе. В практике применяется множество способов оценки рисков, рассматривающих риски под различными ракурсами. Многие из этих способов дают уникальную и полезную информацию, а, следовательно, имеют право на существование. Однако традиционный, хорошо изученный взгляд на риск, используемый в финансовой теории принятия решений в условиях неопределенности, вряд ли применим в контексте стратегического менеджмента, фокусом которого выступают стратегические риски. В теории принятия решений обычно происходит оценка вероятности, направленная на уменьшение неопределенности. Таким образом, фирма, последовательно терпящая примерно одинаковый убыток из года в год, не будет считаться рискованной потому, что результаты её деятельности статичны с низкой или с отсутствием дисперсии. Аналогичным образом, фирма, деятельность которой по прогнозу будет последовательно приносить положительную отдачу, не будет восприниматься как рискованная, даже если эта отдача будет ниже, чем у конкурентов.

Стратегический же подход к риску предполагает, что огромной опасностью для фирмы выступает вероятность того, что её деятельность будет приносить более низкий результат, чем деятельность конкурентов. В конкурентной среде благополучные фирмы добиваются конкурентного преимущества над менее успешными. Использование ресурсов, подкрепленное адекватной и подходящей стратегией, позволяет фирме рассматривать внешние события, или, риски, как преимущества, в то время как конкуренты могут рассматривать их как угрозы бизнесу. Это и будет означать, что компания менее подвержена стратегическому риску, т.е. риску, угрожающему стратегии фирмы. В долгосрочном периоде фирма, которая терпит убытки, пусть даже с низкой дисперсией, обречена на банкротство. Таким образом, к стратегическому риску лучше подходить в терминах относительного результата деятельности фирм среди конкурентов, нежели в терминах дисперсии этого результата относительно прогнозируемого. Чтобы научиться оценивать стратегический риск и вырабатывать способы его избегания, необходимо сформировать концептуальное представление о риске со 
стратегической точки зрения. Ведущие ученые-экономисты ${ }^{29}$ предлагают в качестве точки опоры, противопоставить теорию стратегического риска теории принятий решений в условиях неопределенности и теории финансов.

Отличия от теории принятия решений в условиях неопределенности

Менеджеры воспринимают риск менее точно и вообще иначе, чем это происходит в рамках теории принятия решений [March \& Shapira, 1987]. Boпервых, неопределенность относительно конкретного положительного «исхода» не является самым главным аспектом риска. Во-вторых, риск концептуально определяется не в терминах неопределенности. В-третьих, риск редко сводится к единой конструкции, поддающейся количественному измерению.

1.Риск ассочиируется с потерями. Большинство менеджеров по эмпирическим исследованиям ассоциируют риск с отрицательным результатом. Рискованный выбор для них, это тот выбор, который содержит в себе возможность очень плохого исхода. При этом вероятность того, что фирма получит прибыль, которая сильно выше, чем прибыль конкурентов, не выступает в глазах менеджеров риском. В то время как для теории риска, оперирующей понятием дисперсии, нежелательны, как и экстремальные потери, так и экстремальные выигрыши.

2.Риск ассоциируется с количеством (величиной потенщиальных потерь). Менеджеры определяют риск в терминах величины потерь, нежели в терминах моментного распределения прибыли, или иначе вероятности потерь. Менеджеры ассоциирую риск с провалом десятимиллионного проекта, нежели с потерей десятидолларового проекта.

3.Риск многогранен. Теория принятия решений полагает, что риск идеально может быть выражен в едином, поддающемся количественному измерению, показателю, а именно в вероятности. Менеджеры, оценивая риск, редко сводят его к одному показателю, из-за множественности аспектов стратегического риска.

Отличие от финансового ракурса

Ключевым отличаем в подходе к риску в теории финансов от подхода в стратегическом менеджменте выступает различие в принципах управления этим риском.

1.Рыночный риск и риск фирмы. С финансовой точки зрения риск разделяется на систематический и несистематический. Систематический риск ассоциируется с общим состоянием рынка и экономики. Финансовый подход предполагает, что инвесторы не в состоянии уменьшить систематический риск, потому что он оказывает влияние на все предприятия сразу. При этом стратегический менеджмент говорит о том, что компания в состоянии противодействовать систематическому риску путем заполучения конкурентных преимуществ. Рыночные факторы могут воздействовать на все компании, но степень воздействия зависит от адекватности и качества стратегии, в соответствии с которой фирма ведет свою деятельность. Каким образом, например, риск, связанный с отраслью (из классификации J. Slywotzky и John Drzik), по финансовой теории относится к систематическим и по идее не может быть предотвращен, но с точки зрения менеджмента, адекватная стратегия вполне может с ним справится. Это ещё раз доказывает, что стратегические риски имеют особую природу и требуют иного подхода к своему измерению и предотвращению. Несистематический же риск, с финансовой точки зрения, может быть диверсифицирован грамотно сформированным инвестиционным портфелем, и не является первоочередной проблемой для инвестора. В стратегическом же менеджменте специфический риск

\footnotetext{
${ }^{29}$ Bettis \& Thomas, 1990; Bromiley 1991; Ruefli 1990.

Выпуск \#3, 2007 (C) Электронный журнал Корпоративные Финансы, 2007
} 
выступает самым главным беспокойством. Например, риск, связанный $c$ технологией, может привести к потере клиентов и к глубокому кризису.

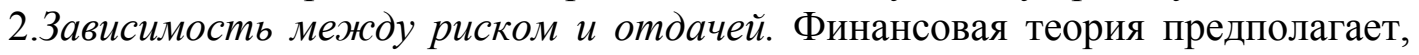
что высокий риск сопряжен с высокой отдачей. Что касается стратегических рисков, то очевидно, что снижение рисков приведет к большей отдаче. И совершенно не факт, что, подвергая себя высокому стратегическому риску, фирма с большей вероятностью получит высокую прибыль.

Miles и Snow предложили рассматривать стратегический риск в трех аспектах: предпринимательском, управленческом и технологическом [Miles, Snow, 1978] . В соответствии с теорией обстоятельств (подход к организационному анализу, предполагающий, что структура и иные особенности организации во многом зависят от факторов внешней среды, используемой компанией технологии и т. д.) каждый из этих аспектов исходит из отношений фирмы с каждым определенным сегментом окружающей среды: предпринимательской среды, потребительской среды, ресурсной среды.

1.Предпринимательский риск, как аспект стратегического риска. В модели стратегического риска предпринимательский риск определяется потенциальными потерями, ассоциируемыми, которые фирма понесет, оперируя в данной бизнес среде, а не в альтернативной.

2.Конкурентный риск, как аспект стратегического риска. В модели стратегического риска конкурентный риск определяется как относительные потери, которые фирма несет, имея данные отношения на товарном рынке со своими потребителями и прочими агентами, по сравнению с тем, что фирма получала бы, имея конкурентное преимущество.

3.Операционный риск, как аспект стратегического риска. Здесь вступают в действие проблемы, технологического характера, которые ассоциируются с построением такой системы, которая позволила бы работать фирме эффективно. Сюда включается выбор подходящих технологий для производства продуктов или услуг, а также, что не менее важно, генерирование и передача информации, контроль и т. д., которые бы обеспечили должное исполнение технологии. Операционный риск в модели стратегического риска измеряется как альтернативные потери фирмы, ассоциирующиеся с данными отношениями фирмы c её ресурсной средой, по отношению с теми, которые бы обеспечили ей эффективную деятельность.

В рис.1 схематично представлен вышеописанный подход к стратегическим рискам. Внешняя среда посылает предприятию риски, которым предприятие противостоит, используя стратегию. При этом стратегия имеет различные аспекты: конкурентный, корпоративный, структурный. Эти риски угрожают стратегии, могут сделать её неадекватной, именно поэтому их определяют как стратегические. Риски возникают из всего многообразия отношений фирмы с окружающей средой на различных уровнях. Именно поэтому менеджеры должны осознавать специфичные аспекты рисков и владеть механизмами борьбы, позволяющими снизить стратегический риск, возникающий из этих аспектов. 


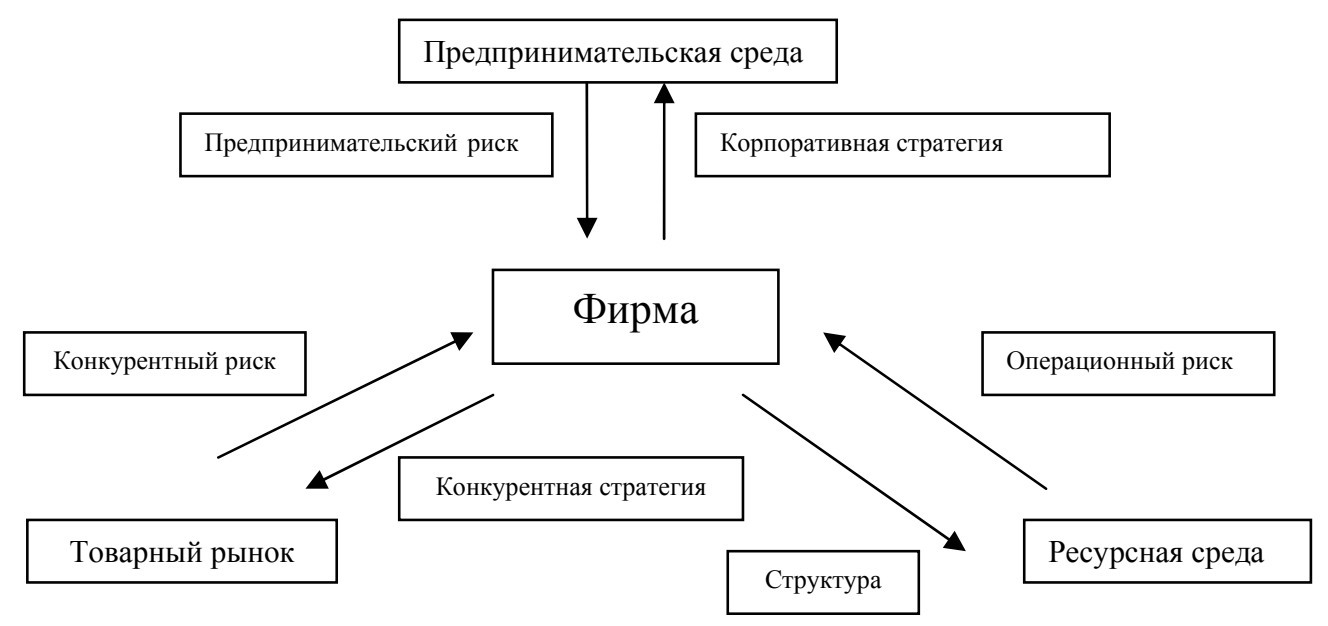

Рис.1 - Формы проявления стратегических рисков

Как было отмечено выше, понятие стратегического риска по своей сути кардинально отличается от общепринятого понятия риска. Традиционно риск определяется через дисперсию. Для стратегического же риска требуется найти иной измеритель. Ввиду многогранности стратегического риска, эта задача становится нетривиальной, и не может быть решена с помощью хорошо изученных подходов типа САРМ.

Ruefli показал, что стратегический риск для конкретной фирмы может быть определен в терминах вероятности потери её положения в рейтинге среди других фирм, относящихся к ней, т.е конкурирующих с ней, производящих товарызаменители [James M. Collins, Timothy W. Ruefli, 1992]. Такой поход опирается на то, что показателем успешности фирмы выступает то, на сколько данная фирма «лучше», чем фирма конкурент, оперирующая в той же бизнес среде. В экономике невозможно судить о качестве результатов деятельности предприятий, если нет возможности соотнести эти результаты с альтернативными, потенциально возможными. Именно исходя из возможности сравнить компании между собой мы выводим такие показатели как средний уровень доходности отрасли и т. д. Поэтому адекватно составленный рейтинг компаний, обычно учитывающий различные показателей «успеха» фирм, является хорошим индикатором рисковой позиции фирмы по стратегическим рискам, то есть позволяет измерить «стратегический риск» фирмы. При этом картину дает не статичное положение компании в рейтинге сейчас, а история перемещений с позиции на позицию. Стратегический риск сопряжен с крупными потерями и его реализация сулит большие проблемы предприятию, если предприятие не способно оградить себя хорошей стратегией и вовремя принять нужные стратегические решения. Перемещение с позиции на позицию по итогам периода в рейтинге компаний как раз и происходит в результате крупных изменений, которые произошли в результате реализации стратегических рисков. Компании, которые сумели вовремя оценить и учесть надвигающийся на горизонте стратегический риск, приобретают конкурентное преимущество и идут вверх по «карьерной лестнице», предприятия, стратегия которых оказалась сломленной под натиском таких серьезных рисков как стратегические, падают вниз. Ruefli предлагает оценить стратегический риск индивидуальной фирмы работающей в системе (отрасли, среде) с помощью математической модели энтропии, с опорой на подход к стратегическому риску, который определяет его через риск потери положения фирмы относительно фирм 
конкурентов. В данном подходе нас не интересует конкретный профиль определенных стратегических рисков, более того, нас не интересует вероятность их наступления. Предметом интереса выступает именно способность фирмы противостоять этим рискам.

Рассмотрим стратегическую систему (набор фирм составляющих отрасль или сегмент отрасли, или же продавцов-конкурентов на каком-то конкретном рынке). Пусть таких фирм будет n. Введем для упрощения анализа предпосылку о том, что все $\mathrm{n}$ фирм постоянно участвуют в деятельности нашего сегмента, однако в принципе можно рассмотреть такую модель, где этого ограничения нет. Наблюдения деятельности системы распределены равномерно во времени. Таким образом, мы имеем матрицу $\mathrm{X}_{\mathrm{nm}}$, характеризующую результаты поведения фирм в период от $\mathrm{t} 0$ до tm. Компонентами матрицы выступают номера позиций, которые фирма $\mathrm{i}$ занимает в каждый момент времени от t0 до tm. $X=\left\{x_{i j} \mid i \in\{1, \ldots, n\}, j \in\{0, \ldots, m\}\right\} \quad$, где і отвечает за номер фирмы, а т за номер наблюдения. Так как в данной матрице содержится информация о том, какое место каждая фирма занимает в каждый период, то для каждой фирмы i возможно сгенерировать матрицу $\Phi_{i}$ элементами которой будут выступать $\Phi_{i j k}$, обозначающими количество раз, когда фирма i переместилась с места $\mathrm{j}$ на место $\mathrm{k}$ за период от t0 до tm. Например, если фирма принадлежит системе из 5 фирм, и мы наблюдаем за деятельностью этой системы в течение 10 периодов, и имеем вектор наблюдений занимаемых позиций фирмой $\mathrm{i}-\{3,4,5,2,1,1,1,1,4,3\}$, то матрица перемещений для фирмы і выглядит следующим образом:

$$
\text { Период } \rightarrow t=\alpha\left[\begin{array}{rrrrrr}
\text { Период } \rightarrow t=\alpha+1 \\
1 & 2 & 3 & 4 & 5 \\
1 & 3 & - & - & 1 & - \\
2 & 1 & - & - & - & - \\
3 & - & - & - & 1 & - \\
4 & - & - & 1 & - & 1 \\
5 & - & 1 & - & - & -
\end{array}\right]
$$

\section{Рис.2 - Матрица перемещений фирмы}

Если все матрицы $\Phi_{i}$, описывающие индивидуальное положение фирмы просуммировать, то получится матрица $\Phi=\left[\sum_{i} \Phi_{i j k}\right]=\Phi_{j k}$, описывающая все перемещения с позиции на позицию для всей системы фирм. Определим условные вероятности с место ј на место $\mathrm{k}: p_{j k}=p_{k \mid j}=\frac{\Phi_{j k}}{\Phi_{j}}$, где $\Phi_{j}$ - сумма элементов в каждом ряде матрицы $\Phi$, описывающая все перемещения в системе, $\Phi_{j k}$ компонент j-ой строки, k-ого столбца. Из полученных элементов $p_{j k}$ составим матрицу, аналогичную по строению матрице $\Phi$, однако компонентами матрицы теперь будут вероятности $p_{j k}$. По построению матрицы $p_{j}=\sum_{k} p_{j k}=1, \quad p_{k}=\sum_{j} p_{j k}=1$, что свидетельствует о двойной стохастичности полученной матрицы. Для примера если $p_{2,1}=0,75$, это означает, что исторически в 3-х из 4-х случаев фирма, находящаяся на второй позиции рейтинга в следующем периоде оказывалась на первом месте. Если бы смоделированный процесс был бы 
рандомен от природы, то эта вероятность равнялась бы $1 / \mathrm{n}$, где $\mathrm{n}-$ количество обследуемых фирм. Если же процесс наоборот был бы полностью предопределен, то только одна вероятность $p_{j k}$ в каждом столбце и строке равнялась бы единице. Таким образом, распределение $p_{j k}$ вероятностей содержит информацию об обычных перемещениях в системе. Далее предлагается ввести функцию энтропии, которая позволяет оценить количество информации, содержащейся в предшествующих наблюдениях перемещений фирм в системе, и в основе которой лежит вероятностное распределение, описывающее поведение системы.

(1) $H_{K \mid J}=-\sum_{j} \sum_{k}\left[\frac{p_{k \mid j} \ln \left(p_{k \mid j}\right)}{n}\right], \quad p_{j k} \geq 0, \sum_{j} p_{j k}=\sum_{k} p_{j k}=1$,

$H_{K \mid J}$ описывает количество информации содержащейся в прошлых наблюдениях положения фирм в системе. Однако эта величина безразмерная и поэтому её величину трудно интерпретировать. Поэтому предлагается провести нормировку на $H_{K \mid J}^{*}$, обозначающую энтропию гипотетической максимально неопределенной системы из $\mathrm{n}$ фирм и т наблюдений.

(2) $H_{K \mid J}^{*}=-\sum_{j} \sum_{k}\left[\frac{\ln (1 / n)}{n \times n}\right]=\ln n$

В результате нормировки получаем:

(3) $H_{(K \mid J) r e l}=\frac{H_{K \mid J}}{H^{*}{ }_{K \mid J}}$,

$H_{(\kappa \mid J) r e l}$ выражает относительную неопределенность системы. Другими словами, полученный результат обозначает то преимущественное количество информации, которое мы имеем о системе, проведя анализ наблюдений её деятельности, по сравнению с тем количеством информации, которым мы бы обладали, если бы знали только количество фирм, действующих в системе. Когда вероятность каждого перемещения в системе равна $1 / \mathrm{n}$, её энтропия максимальна, и систему можно назвать рандомной.

В первоначальном упрощенном виде все перемещения в системе неявно предполагались равнозначными, то есть имеющими одинаковую важность. Однако это не совсем реалистично. В действительности же аналитики считают, что при прочих равных, например, перемещение с первого на пятое место имеет более важный вклад в стратегическую неопределенность системы, чем скажем перемещение с первой на вторую позицию. Соответственно модель стоит дополнить с учетом этих предпосылок. Усовершенствованная модель отражает два элемента неопределенности ситуации:

1.Элемент, относящийся к вероятности наступления события (перемещения с позиции на позицию)

2.Элемент, относящийся к серьезности сдвига, то есть отражающий, на сколько сильно фирма набрала или потеряла позицию

Хорошим инструментом для учета обоих условий выступает функция взвешенной энтропии ${ }^{30}$. Вводим в модель веса $w_{j, k}=\alpha|k-j|+\beta, \forall j<k$, тем самым, предполагая, что значимость сдвигов прямо пропорциональна количеству потерянных или набранных позиций. Взвешенная функция средней условной энтропии, которая отражает стратегическую неопределенность системы, может быть определена следующим образом:

\footnotetext{
${ }^{30}$ Guiasu 1977; Theil 1980
}

Выпуск \#3, 2007 
(4) $H W_{K \mid J}=-\sum_{j} \sum_{k}\left[\frac{w_{j k} p_{k \mid j} \ln \left(p_{k \mid j}\right)}{n}\right], \quad p_{j k} \geq 0, \sum_{j} p_{j k}=\sum_{k} p_{j k}=1$

Определение максимума взвешенной условной энтропии является более сложной процедурой, так как для этого нужно решить задачу математического программирования:

$$
\begin{aligned}
& \text { (5) } H W_{K \mid J}^{*}=M A X H W_{K \mid J}=M A X-\sum_{j} \sum_{k}\left[\frac{w_{j k} p_{k \mid j} \ln \left(p_{k \mid j}\right)}{n}\right] \\
& p_{j k} \geq 0, \sum_{j} p_{j k}=\sum_{k} p_{j k}=1
\end{aligned}
$$

Относительная взвешенная средняя условная энтропия $H W^{*}{ }_{K \mid J}$ может быть выражена как:

(6) $H W_{(K \mid J) r e l}=\frac{H W_{K \mid J}}{H W_{K \mid J}^{*}}$,

Риск системы ассоциируется именно с перемещением с более высокой позиции на более низкую позицию $(k>j)$, таким образом, $N W R_{K \mid J}$, обозначающий систематический риск системы может быть выражен как:

(7) $H W R_{K \mid J}=-\sum_{j} \sum_{k>j}\left[\frac{w_{j k} p_{k \mid j} \ln \left(p_{k \mid j}\right)}{n}\right]$,

(8) $H W R_{(K \mid J) r e l}=\frac{H W R_{K \mid J}}{H W^{*}{ }_{K \mid J}}$

$H W R_{(K \mid J) r e l}$ дает возможность измерить относительный риск системы, связанный с потерей позиций фирмами, оперирующими в исследуемой нами системе. Более того, так как показатель нормализован, то он дает возможность сравнивать системы разной величины.

Вышеописанная модель описывает способ измерить стратегический риск условной системы, однако, изначальная цель была описать стратегический риск индивидуального предприятия, живущего в этой системе. Уравнение $p_{j k}=p_{k \mid j}=\frac{\Phi_{j k}}{\Phi_{j}}$, может быть использовано для описания взаимоотношения между одной фирмой и всей системой. Для этого его надо немного трансформировать. Таким образом, ожидаемая оценка общей взвешенной информации о перемещениях одной фирмы относительно перемещений системы с позиции $\mathrm{j}$ на $\mathrm{k}$ может быть описана следующей функцией:

$$
\text { (9) } h w_{i K \mid J}=-w_{j k}\left[\frac{\Phi_{i j k}}{\Phi_{j}}\right] \ln \left(p_{k \mid j}\right) \text {, }
$$

Для перемещения с позиции $j$ на $k$, взвешенная энтропия для фирмы $i$ это информация $\left(=\ln \left(p_{k \mid j}\right)\right)$ о величине потерь $\left(=w_{j k}\right)$, которые принадлежат одной фирме i $\left(=\frac{\Phi_{i j k}}{\Phi_{j}}\right)$.

Если просуммировать все перемещения фирмы $i$ в системе получаем, что общая неопределенность, приходящаяся на долю фирмы $i$, может быть определена как:

$$
\text { (10) } H W R_{i(K \mid J)}=-\sum_{j} \sum_{k>j}\left[\frac{\Phi_{i j k}}{\Phi_{j}} \frac{w_{j k} \ln \left(p_{k \mid j}\right)}{n}\right] \text {, }
$$


Далее нормализуем стратегический риск фирмы $i$ :

(11) $H W R_{i(K \mid J) r e l}=\frac{H W R_{i(K \mid J)}}{H W_{K \mid J}^{*}}$

Таким образом, наша конечная функция, измеряющая величину индивидуального стратегического риска фирмы в системе, по своему смыслу соответствует данному ранее определению Ruefli стратегического риска, которое говорит что, стратегический риск для конкретной фирмы может быть определен в терминах вероятности потери её положения в рейтинге среди других фирм, относящихся к ней, т.е. конкурируюших с ней, производящих товары-заменители и m.д. Выражение (11) отражает:

1.Шанс именно «потери позиции»

2.Величину вероятности «потери позиции»

3.Количество «потерь позиции»

Такой стандартный подход к измерению совокупного стратегического риска, ассоциирующегося с индивидуальной фирмой, представляет собой ex post измерение риска, так как основывается на исторической информации прошлых периодов. Мы как бы измеряем способность фирмы бороться со своими стратегическими рисками, которая сложилась исторически. Более того, мы определяем эту способность относительно способности всей отрасли или сегмента. Такая оценка стратегического риска ex post в принципе может быть использована для оценки поведения фирмы ex ante. Мы как бы полагаемся на память нашего предприятия и просто экстраполируем её прошлое поведение на будущие периоды. Можно сказать, что существует связь между исторически выявленной способностью фирмы бороться с её стратегическим риском и результатами её будущей деятельности.

\section{Список литературы}

1.Chandler A. D (1962), Strategy and structure, Cambridge, MA: MIT Press, 1962

2.Drzik, J., Slywotzky, A.J. (2005), Countering the biggest Risk of All, Harvard Business Review 4

3.Ellen Earle Chaffee (1985), Three Models of Strategy, The Academy of Management Review, Vol. 10, No.1. (Jan., 1985), pp. 89-98.

4.Heini Ikavalko, Petri Aaltonen (2001), Middle Managers' Role in Strategy Implementation, 17-th EGOS Colloquium, July 5-7, 2001, Lyon, France.

5.Hofer M.L. (1973), Some preliminary research on patterns of strategic behavior, Academy of Management proceeding, 1973

6.James M. Collins, Timothy W. Ruelfi (1992), Strategic Risks: An Ordinal Approach. Management Science, Vol 38, \#12 (Dec. 1992), pp 1707-1731.

7.Lenz R. Environment, strategy, organization, structure and performance (1980), Strategic Management Journal, 1980b

8.Lorange P., Vancil R.F., How to design a strategic business planning system, Harvard business review, 1978,3

9.March, J. G., Shapira, Z. (1987), Managerial perspectives on risk and risk taking, Management Science, 33 (11)

10. Miles, R. E., Snow, C.C. (1978), Organizational strategy, structure and process, New York: McGraw Hill.

11. Simons R. A Note on Identifying Strategic Risks(1999), Harward Business School Review, November. P.1. 